\title{
Alcohol drinking patterns and risk of diabetes: a cohort study of 70,551 men and women from the general Danish population
}

\author{
Charlotte Holst $^{1}$ • Ulrik Becker ${ }^{1,2}$ • Marit E. Jørgensen ${ }^{1,3}$ • Morten Grønbæk ${ }^{1}$ • \\ Janne S. Tolstrup ${ }^{1}$
}

Received: 10 February 2017 / Accepted: 1 June 2017 /Published online: 27 July 2017

(C) Springer-Verlag GmbH Germany 2017

\begin{abstract}
Aims/hypothesis Alcohol consumption is inversely associated with diabetes, but little is known about the role of drinking patterns. We examined the association between alcohol drinking patterns and diabetes risk in men and women from the general Danish population.

Methods This cohort study was based on data from the Danish Health Examination Survey 2007-2008. Of the 76,484 survey participants, 28,704 men and 41,847 women were eligible for this study. Participants were followed for a median of 4.9 years. Self-reported questionnaires were used to obtain information on alcohol drinking patterns, i.e. frequency of alcohol drinking, frequency of binge drinking, and consumption of wine, beer and spirits, from which we calculated beverage-specific and overall average weekly alcohol intake. Information on incident cases of diabetes was obtained from the Danish National Diabetes Register. Cox proportional hazards model was applied to estimate HRs and 95\% CIs.

Results During follow-up, 859 men and 887 women developed diabetes. The lowest risk of diabetes was observed at 14 drinks/week in men (HR 0.57 [95\% CI 0.47, 0.70]) and at 9 drinks/week in women (HR 0.42 [95\% CI 0.35, 0.51]),
\end{abstract}

Electronic supplementary material The online version of this article (doi:10.1007/s00125-017-4359-3) contains peer-reviewed but unedited supplementary material, which is available to authorised users.

Janne S. Tolstrup

jst@niph.dk

1 National Institute of Public Health, University of Southern Denmark, Øster Farimagsgade 5A, 2. Floor, 1353 Copenhagen, Denmark

2 Gastro Unit, Medical Division, Copenhagen University Hospital Hvidovre, Hvidovre, Denmark

3 Clinical Epidemiology, Steno Diabetes Center, Gentofte, Denmark relative to no alcohol intake. Compared with current alcohol consumers consuming $<1$ day/week, consumption of alcohol on 3-4 days weekly was associated with significantly lower risk for diabetes in men (HR 0.73 [95\% CI 0.59, 0.94]) and women (HR 0.68 [95\% CI 0.53, 0.88]) after adjusting for confounders and average weekly alcohol amount.

Conclusions/interpretation Our findings suggest that alcohol drinking frequency is associated with risk of diabetes and that consumption of alcohol over 3-4 days per week is associated with the lowest risk of diabetes, even after taking average weekly alcohol consumption into account.

Keywords Alcohol $\cdot$ Alcohol consumption $\cdot$ Alcohol drinking patterns $\cdot$ Basic science $\cdot$ Diabetes $\cdot$ Drinking patterns $\cdot$ Epidemiology $\cdot$ Human $\cdot$ Risk $\cdot$ Type 2 diabetes mellitus

\section{Abbreviation \\ DANHES Danish Health Examination Survey}

\section{Introduction}

Epidemiological studies have consistently suggested that light to moderate alcohol consumption is associated with a lower risk for diabetes compared with abstention in men [1-5] and women [1-6]. Heavy consumption is, on the other hand, associated with a risk equal to [7] or greater than [8] that of abstainers. However, results from the few prospective studies which have examined the role of drinking patterns, such as frequency, binge drinking (i.e. consuming large amounts of alcohol per occasion) and beverage type, in relation to type 2 diabetes are inconsistent. Frequent alcohol consumption, compared with alcohol consumption concentrated over a few 
days, has been suggested to convey lower risks of diabetes, even if the alcohol amount consumed per drinking day is low, implying that frequency is more important than amount $[9$, 10]. However, other studies have shown that frequent alcohol consumption is not associated with lower risks of diabetes when adjusting for total alcohol amount $[8,11]$. Another area that needs to be further addressed is whether the potential beneficial effect of alcohol on the risk of diabetes pertains to specific beverage types. Although wine has been suggested to have particularly protective effects in relation to type 2 diabetes $[12,13]$, epidemiological studies are inconclusive when it comes to the specific effects of beer and spirits [13-15]. In addition, binge drinking has been associated with an increased risk of type 2 diabetes among men [8, 16] and women [17], while others have reported a tendency of reduced risk associated with binge drinking [18].

Diverging findings from previous studies may have several explanations: for example, using different definitions of alcohol drinking patterns and various combinations of alcohol drinking frequency and amount in relation to the risk type 2 diabetes makes it difficult to compare studies. However, despite differences in methods, the results imply the importance of alcohol drinking patterns in addition to quantity consumed. To address these issues, we investigated the association between alcohol drinking patterns and the risk of diabetes among men and women from the general Danish population.

\section{Methods}

The Danish Health Examination Survey Data from the Danish Health Examination Survey (DANHES) from 2007 to 2008 was used [19]. DANHES was conducted in 13 of 98 municipalities in Denmark, where all citizens in the participating municipalities aged $\geq 18$ years were invited to complete a self-reported questionnaire comprising items on lifestyle, health and morbidity. In 12 municipalities, an internet-based questionnaire was used, whereas a paper-based questionnaire was used in the remaining municipality. Overall 538,497 people were invited and 76,484 participated (14\%). The personal identification number - a unique ten-digit number assigned to each Danish citizen - enabled linkage to Danish nationwide registries from where information on social factors, morbidity and mortality was obtained.

Alcohol drinking patterns Lifetime abstainers were defined as individuals, who reported never drinking alcohol, and current abstainers were defined as individuals with a history of drinking alcohol, but who had not drunk alcohol within the last year. Respondents who had been drinking alcohol within the last year were asked about their alcohol drinking pattern. Information on frequency of alcohol drinking was reported as: $<1$ day/week; 1-2 days/week; 3-4 days/week; or 5-7 days/ week. Participants were asked about frequency of binge drinking ( $\geq 5$ beverages/occasion), which was reported as: never; $<1$ day/week; 1 day/week; or $>1$ day/week. Furthermore, participants were asked to report, in drinks, their average daily consumption of wine, beer and spirits during the seven weekdays. One Danish standard drink corresponds to $12 \mathrm{~g}$ ethanol. Based on average weekly alcohol consumption of specific beverage types, we calculated beverage-specific and the overall average weekly alcohol amount. The beverage-specific alcohol amount was coded as: $<1$ drink/week; 1-6 drinks/week; $\geq 7$ drinks/week (women); 7-13 drinks/week (men); or $\geq 14$ drinks/week (men). Last, participants were asked whether their consumption of alcohol within the last 5 years had been decreasing, increasing or stable.

Diabetes Information on diabetes during follow-up was obtained from the Danish National Diabetes Register, which comprises all incident cases of diabetes diagnosed in Denmark from 1991 and onwards [20]. The registry uses five diagnostic criteria: (1) hospitalisation with a diagnosis of diabetes according to the ICD 8th or 10th Revisions (ICD-8 codes 249 or 250; ICD-10 codes E10-14, H36.0 or O24 [excluding O24.4], www.who.int/classifications/icd/en/) obtained from the Danish National Patient Registry; (2) registration of chiropody (coded for diabetes) in the Danish National Health Service Register; (3) registration in the Danish National Health Service Register with measurement of blood glucose five or more times within 365 days; (4) two or more annual measurements of glucose during a 5 year period; or (5) registration in the Danish National Prescription Registry with prescription of insulin or oral glucoselowering medication on at least two occasions. If one of these criteria is met, an individual is registered as having diabetes. The register does not distinguish between type 1 and type 2 diabetes. Participants were followed up for diabetes in the register to December 2012.

Covariates Based on the literature, we considered the following as confounders: age, sex, BMI (calculated as $\mathrm{kg} / \mathrm{m}^{2}$ ) as a linear and squared term, education, smoking status (never smoker, former smoker, occasional smoker or daily smoker), diet, leisure-time physical activity, hypertension (current or previous) and family history of diabetes. All covariates except age and sex were based on self-report in DANHES. Level of school education was defined according to the International Standard Classification of Education, combining ongoing or completed school and vocational education and divided into: $<10$ years; $10-12$ years; $13-14$ years; or $\geq 15$ years. Information on diet was based on five questions about intake of: (1) fibre-rich bread or grain; (2) boiled, fried or baked vegetables; (3) salad; (4) fruit; and (5) fish at dinner. For each of diet questions $1-4$, respondents were coded as infrequent eaters if they ate the respective food never/very infrequently, 
$<1$ time/week, 1 time/week, or a couple of times/week. Respondents were coded as frequent eaters if they ate the food almost every day, or every day/more than once a day. For diet question 5, respondents were categorised as infrequent eaters if they ate fish for dinner never/very infrequent or $<1$ time/week. Otherwise they were reported as frequent eaters. Leisure-time physical activity during the last year was assessed based on a measure developed by Saltin and Grimby [21], with the following response categories: (1) vigorous (strenuous activities usually involving competition or endurance training performed regularly or several times a week); (2) moderate (exercise, endurance training or heavy gardening for at least $4 \mathrm{~h}$ a week); (3) light (walking, cycling or other light activities for a minimum of $4 \mathrm{~h}$ a week); or (4) inactive (reading, TV watching or other sedentary activities). Respondents were categorised as having a family history of diabetes if they reported having a mother, father and/or full brothers and sisters with diabetes.

Final study population We excluded participants with a diagnosis of diabetes at baseline (date of participation in DANHES) by linkage to the Danish National Diabetes Register $(n=3079)$ or if they reported a history of diabetes in DANHES $(n=543)$. Women who were pregnant at baseline or had given birth within the last 6 months from baseline were excluded $(n=894)$, based on the assumption that women reduce their alcohol consumption during pregnancy. Furthermore, we excluded participants with no information about whether they had been drinking alcohol within the last year $(n=783)$, how often they consumed alcohol $(n=460)$ or how often they binge drank $(n=174)$. The final study population comprised 70,551 individuals (28,704 men and 41,847 women).

Statistical analyses Through linkage to the Danish Civil Registration System we obtained information concerning time of death and emigration. Participants were followed from baseline until diagnosis of diabetes $(n=1746)$, emigration $(n=454)$, death $(n=1254)$ or 29 December 2012 ( $n=67,097)$, whichever occurred first. The total observation time comprised 342,349 person-years, and both men and women were followed for a median of 4.9 years ( range $=0.0$ 5.8 years).

To account for missing values for potential confounders, we performed multiple imputation by chained equations with 20 repetitions. The imputation model included variables that were hypothesised to predict missing information (age, sex and diabetes). The numbers of missing values were 1944 (physical activity), 61 (smoking), 152 (vegetables), 182 (salad), 182 (fruit), 192 (fibre-rich bread or grain), 194 (fish), 1953 (beer, wine and spirits), 2460 (BMI), 4110 (school education), 4682 (hypertension) and 5815 (family history of diabetes).
Data were analysed by means of the Cox proportional hazards regression model to estimate HRs and $95 \%$ CIs for the risk of developing diabetes. Age (in days) was used as the underlying time axis, which ensured maximal adjustment for confounding by age. All analyses were carried out separately for men and women, as the literature suggests sex differences in the relationship between alcohol consumption and risk of type 2 diabetes [6, 22]. Further, preliminary analyses of data suggested interactions between sex and several of the independent variables and the outcome. The assumption of proportional hazards was tested graphically and in statistical tests and the assumption was found to be fulfilled for all models. Curvilinear associations between alcohol variables and diabetes were examined by numerical recoding of the median value within categories of the examined alcohol variables and adding these as continuous variables to the models. Lifetime and current abstainers were excluded from the analysis testing for trend. In the analyses of frequency of binge drinking and beverage type, abstainers were not included in the tests.

To examine the continuous measure of average weekly alcohol amount, a restricted cubic spline model, using three knots (values were 1, 8 and 22 for men, and 0,4 and 13 for women), was fitted [23]. A nested log likelihood test was performed to compare a model with three knots with a model with four knots. There was no statistically significant difference between the two in the prediction of the relationship between alcohol and risk for diabetes; hence the model with three knots was applied. The reference level was set at 0 drinks/week (comprising individuals consuming 0 drinks/ week, current abstainers and lifetime abstainers). As cubic spline models are sensitive to outliers, we excluded participants with an average weekly alcohol amount above the 99th centile, corresponding to 40 drinks/week in men and 28 drinks/week in women.

To test for interaction between drinking frequency and alcohol amount we used a nested log likelihood test where we compared a model containing the variables as single terms with a model including the interaction terms.

Because BMI is most likely both a confounder and a mediator in the association between alcohol consumption and type 2 diabetes [2], we conducted multivariate analyses where BMI was not included as a covariate in order to examine how this affected the results.

We performed sensitivity analyses where the effects of average weekly alcohol amount and frequency of alcohol consumption on the risk of diabetes were tested. First, we excluded participants who had reduced or increased their alcohol consumption within the last 5 years of baseline. Second, as the Danish National Diabetes Register does not contain information about diabetes type, and incidence of type 2 diabetes rises from middle life, we performed a sensitivity analysis based on participants aged 40 years or above at baseline. Furthermore, as suggested by others [24], a sensitivity 
analysis was performed where criteria 3 (measurement of blood glucose five or more times within 365 days) and 4 (two or more annual measurements of glucose during a 5 year period) in the Danish National Diabetes Register were not used as indicators of diabetes. This was because of a suspicion that a large proportion of the individuals included in the Danish National Diabetes Register based on these criteria do not have clinically diagnosed diabetes [24].

The significance level was set to a $p$ value of 0.05 (twosided). Analyses were performed using SAS Software Enterprise Guide version 5.1, SAS Institute, Cary, NC, USA and STATA version 14.2, StataCorp TX, USA. All study participants gave their informed consent to the research, and the publication of results was approved by the Danish Data Protection Agency.

\section{Results}

\begin{abstract}
Alcohol drinking characteristics of the study population Table 1 shows the characteristics of the study population according to frequency of alcohol drinking. Among alcohol drinkers, the median weekly alcohol amount was eight and four drinks in men and women, respectively. The median weekly alcohol amount and the fraction of weekly binge drinkers increased with increasing frequency of alcohol drinking. The distribution of the consumption of beer, wine and spirits differed across the groups of alcohol drinking frequency; beer constituted about half of the total amount of alcohol consumed in men drinking < 1 day/week and 1-2 days/week, while wine comprised half of the total alcohol intake among men who drank alcohol on 3-4 days/week or more. In women, wine constituted more than half of the total alcohol intake across all frequency groups, with a higher percentage concurrently with increasing alcohol drinking frequency.
\end{abstract}

Average weekly alcohol amount Among the 70,551 study participants, 859 men and 887 women developed diabetes during follow-up, corresponding to an incidence rate of diabetes of 619 and 436 per 100,000 person-years, respectively ( $p<0.0001$ for sex difference).

The restricted cubic spline curves of the continuous alcohol amount variable confirmed a U-shaped association between average weekly alcohol amount and diabetes risk for men (Fig. 1a) and women (Fig. 1b). The lowest risk of diabetes was observed at 14 drinks/week in men (HR 0.57 [95\% CI $0.47,0.70]$ ) and 9 drinks/week in women (HR 0.42 [95\% CI $0.35,0.51]$ ). We investigated the association between average weekly alcohol amount up to 40 drinks/week in men and 28 drinks/week in women with the risk of developing diabetes. Within these levels of alcohol consumption, the risk of diabetes did not exceed the risk of the reference level.
Frequency of alcohol drinking Table 2 shows the relative risks of diabetes according to frequency of alcohol drinking. When adjusting for confounders and average weekly alcohol amount, only consumption of alcohol 3-4 days/week was significant, with HR 0.73 (95\% CI 0.59, 0.94) and 0.68 (95\% CI $0.53,0.88)$ in men and women, respectively. Among women, lifetime abstainers had a higher risk of diabetes (HR 1.77 [95\% CI 1.25, 2.51]), when compared with consumption $<1$ day/week (Table 2).

We examined the absolute risks of diabetes by the joint effect of frequency of alcohol drinking and average weekly alcohol amount (Table 3). When adjusting for confounders, men's consumption of $7-13$ drinks or $\geq 14$ drinks over 34 days/week was associated with HR 0.72 (95\% CI 0.54 , 0.96 ) and 0.62 (95\% CI 0.43, 0.88), respectively. Further, men consuming $\geq 14$ drinks over 5-7 weekdays also had a lower risk of diabetes (HR 0.75 [95\% CI 0.61, 0.92]). Among women, consumption of 1-6 drinks (HR 0.64 [95\% CI $0.45,0.93]$ ) and $\geq 7$ drinks (HR 0.72 [95\% CI 0.55, 0.95]) consumed over 3-4 days/week was associated with a lower risk of diabetes. No significant associations were found for other combinations of frequency of alcohol drinking and average weekly alcohol amount.

No statistically significant interaction was found between drinking frequency and alcohol intake on risk of diabetes (men, $p=0.533$; women, $p=0.408$ ).

Cubic splines models of the association between alcohol intake and diabetes did not change noticeably when BMI was excluded from the model. However, some of the estimates for frequency of alcohol drinking and diabetes became significant; e.g. the HRs for diabetes in men consuming alcohol on 1-2 days/week and 5-7 days/week were 0.77 (95\% CI 0.62, $0.94)$ and 0.71 (95\% CI 0.57, 0.89). The corresponding HRs for women were 0.74 (95\% CI 0.62, 0.89) and 0.65 (95\% CI $0.49,0.85)$ (data not shown).

Frequency of binge drinking and beverage type We found no clear evidence of an association between binge drinking and risk of diabetes (electronic supplementary material [ESM] Table 1). ESM Table 2 shows the results of the analyses of average weekly intake of beer, wine and spirits on the risk of diabetes. Consumption of 1-6 drinks of beer/week was significantly associated with a lower risk of diabetes in men (HR $0.79[95 \%$ CI $0.67,0.94])$. In addition, men who consumed $\geq 7$ drinks of wine/week had around a $30 \%$ lower risk of diabetes compared with men drinking $<1$ drink of wine/week. We found no statistically significant association between average weekly alcohol amount of spirits and diabetes in men. In women, however, drinking $\geq 7$ drinks of spirits/week was associated with HR 1.83 (95\% CI 1.29, 2.58) compared with women drinking $<1$ drink of spirits/week. Also, women drinking one 


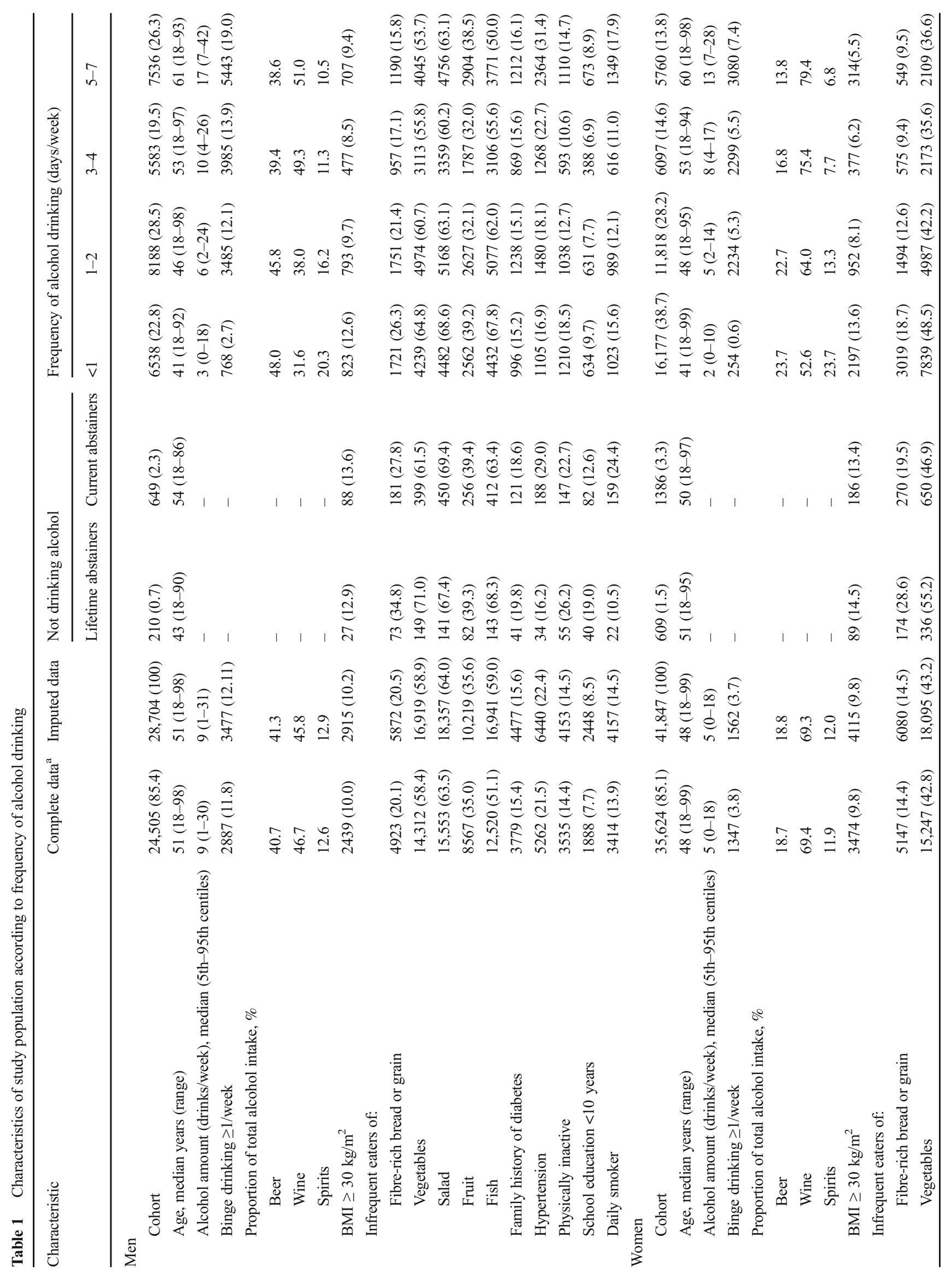




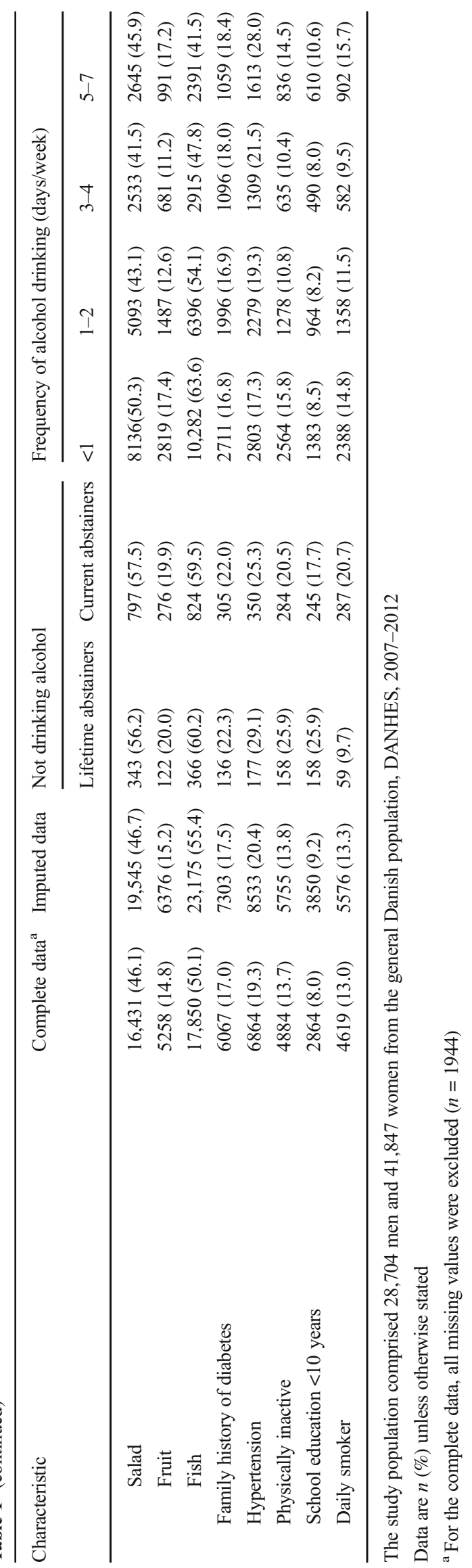



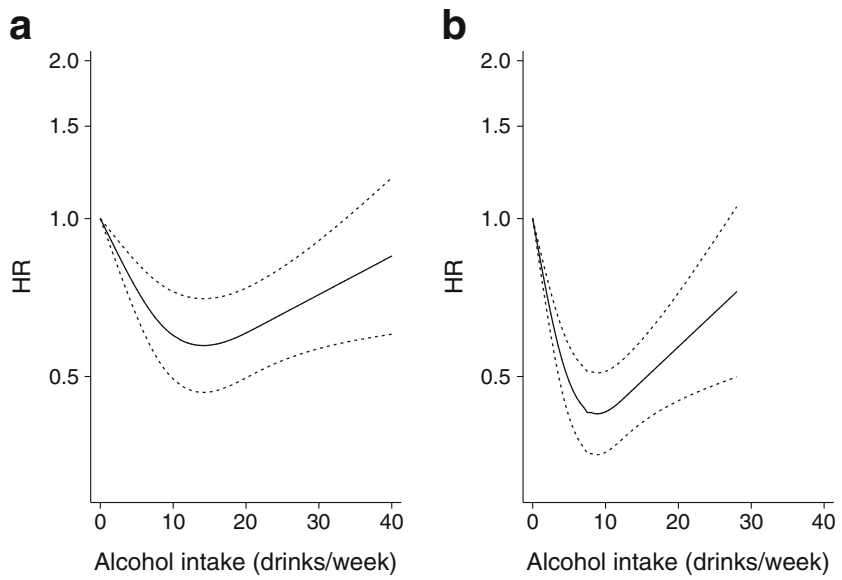

Fig. 1 Risk for diabetes in 28,704 men (a) and 41,847 women (b) from the general Danish population according to average weekly alcohol amount, DANHES, 2007-2012. Solid lines are multivariable-adjusted HRs and dashed lines indicate $95 \%$ CI derived from a restricted cubic spline regression. HRs (95\% CIs) are shown on a natural log scale. The graphs are truncated at the 99th centiles. Age (in days) is the underlying time axis. Adjusted for BMI (linear and squared effect), diet, family history of diabetes, hypertension, physical activity, school education and smoking

drink or more of wine/week had a lower risk of diabetes when compared with women drinking $<1$ drink of wine/week.

Sensitivity analyses Sensitivity analyses were performed in which we tested the effects of average weekly alcohol amount and frequency of alcohol consumption on the risk of diabetes (data not shown). First, we excluded participants who had reduced $(n=14,711)$ or increased $(n=7302)$ their alcohol consumption within the last 5 years; this did not change the estimates noticeably. Second, excluding participants under the age of 40 did not change the results. Last, we omitted criteria 3 and 4 in the Danish National Diabetes Register as indicators of diabetes, as suggested by others [24]; this did not change the estimates noticeably.

\section{Discussion}

Among 28,704 men and 41,847 women from the general Danish population, 859 men and 887 developed diabetes during follow-up. We found that light to moderate alcohol consumption was associated with a lower risk of diabetes, relative to no alcohol consumption. Our results indicate that frequent alcohol consumption is associated with the lowest risk of diabetes, even after taking average weekly alcohol consumption into account.

Our findings should be interpreted in the context of the existing literature. In the present study, we observed the lowest risks of diabetes at 14 and 9 drinks/week in men and women, respectively. Other epidemiological studies have suggested that consumers of moderate levels of alcohol have a lower risk of type 2 diabetes compared with non-drinkers [4, 6, 25]. In this study, frequent alcohol consumption, compared with less frequent consumption, was associated with a lower risk of diabetes. Previously, frequent consumption has been reported to convey the lowest risk of diabetes, even though the amount of alcohol consumed per drinking occasion is low $[9,10]$. However, infrequent drinkers with low average alcohol consumption are shown to drink more during drinking days and have unfavourable risk factor profiles in relation to cardiovascular disease and mortality when compared with more frequent drinkers [26]. This suggests that confounding may contribute to the favourable associations often associated with moderate alcohol consumption and high drinking frequency. Furthermore, alcohol amount consumed per drinking occasion has been proposed to be more important in relation to risk of type 2 diabetes than drinking frequency [8]. In this study, we did not find evidence of an association between binge drinking and risk of diabetes; this could be because of low statistical power, as few participants, especially women, binge drank at least once a week. Previously, conflicting results have been reported showing that binge drinking increases the risk of type 2 diabetes in men, but not women $[8,10,11,16]$, and, vice versa, doubling the risk of diabetes in women but with no association in men [17]. The use of different definitions of binge drinking across studies and different drinking cultures across cultures contributes to the conflicting results. Regarding beverage type, our analyses suggest a lower risk of diabetes associated with a moderate to high intake of wine and, among women, a higher risk of diabetes associated with a high intake of spirits. The association between wine consumption and diabetes is in line with findings from a meta-analysis based on 13 prospective studies, in which wine consumption was associated with a significantly lower risk of type 2 diabetes: $20 \%$ risk reduction at 20-30 g pure alcohol/day [12]. One possible biological explanation of the protective effect of wine is that polyphenols, natural phytochemical compounds found in red wine, may exert beneficial effects on blood glucose control, and thereby lowering the risk of type 2 diabetes [27].

This study has several important strengths. First, the large study population consisted of men and women with a wide age range from the general population. In addition, alcohol drinking patterns were assessed through detailed questions. We were able to distinguish lifetime from current abstainers, an important step as the latter might have given up alcohol drinking because of health problems, whereas other motives can be speculated for lifetime abstainers. Often former drinkers are included in the abstainer reference group, which might 


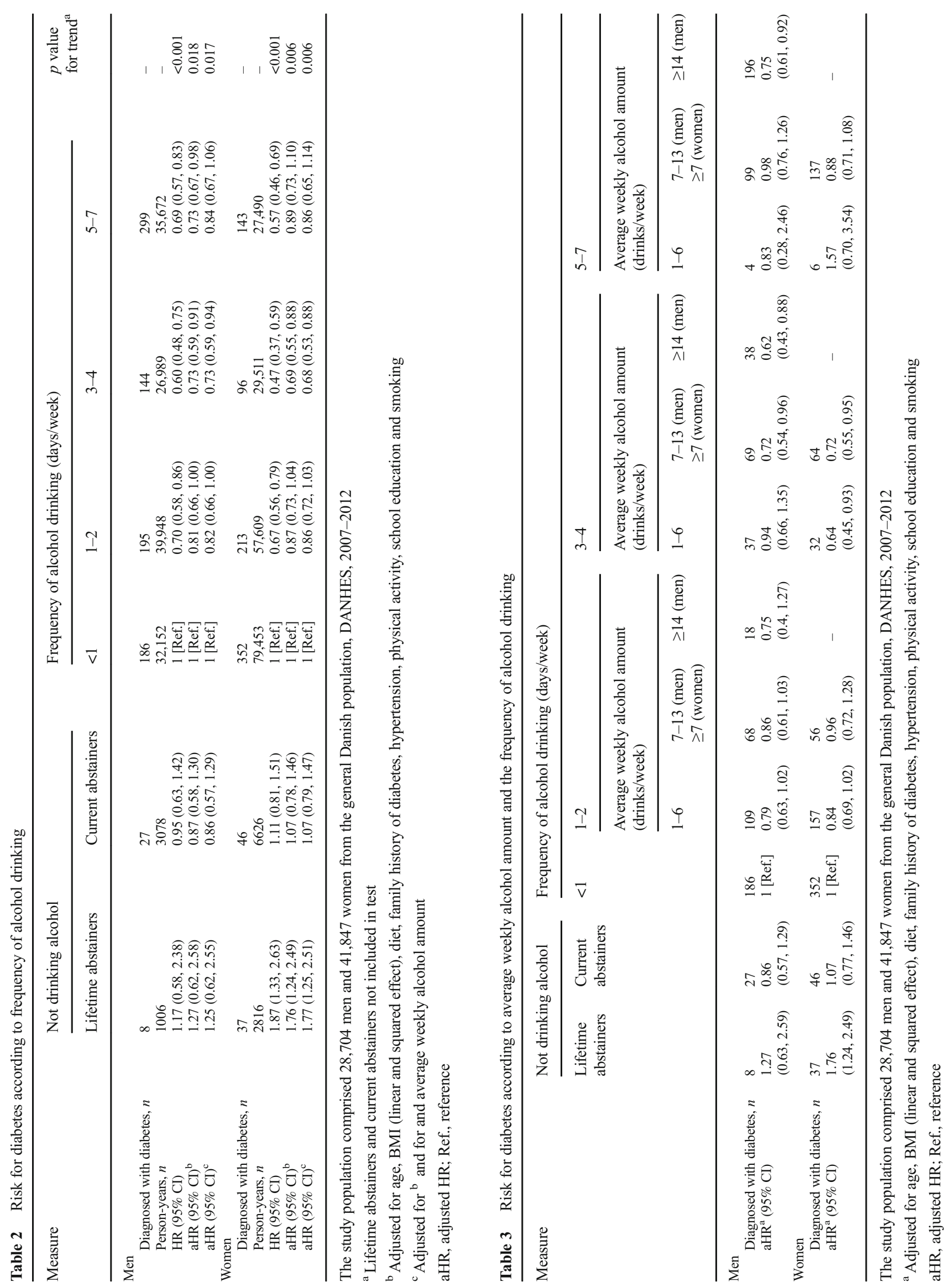


bias drinking risk downward, thereby magnifying the appearance of health benefits associated with low-level drinking [28]. Linkage to national registers ensured precise follow-up. We were also able to adjust for multiple potential confounding variables, such as sex, age, BMI, dietary factors, family history of diabetes, hypertension, physical activity, school education and smoking, in our analysis. Last, it is a strength that the results did not change in the sensitivity analysis we performed.

This study has limitations that must be considered in the interpretation of the findings. First, some misclassification of drinking patterns may have occurred, as alcohol consumption is generally underreported. However, when participants are asked about intake of beer, wine and spirits separately, the most realistic levels of intake are reported [29]. In addition, information about alcohol drinking pattern was collected prior to diagnosis of diabetes and we therefore expect potential misclassification to be non-differential and not a concern in the interpretation of the results. Second, a small number of cases of diabetes were observed in participants who drank $\geq 7$ drinks/week of beer or spirits, which affected the statistical power of these analyses, including the testing for trend. Third, alcohol drinking patterns were assessed at a single point, despite the fact that most individuals change alcohol drinking patterns over time. We cannot therefore rule out that some participants changed drinking patterns during follow-up. Fourth, we were not able to distinguish between type 1 and type 2 diabetes. Sensitivity analysis excluding participants younger than 40 years did not change our results and, although one-third of people with type 1 diabetes are diagnosed after age 30 years, the majority of individuals identified with diabetes during follow-up most likely had type 2 diabetes. Last, information on diet, which is a strong risk factor for type 2 diabetes, was based on dichotomised measures of frequency of intake of five common food items. This measure might be too simple to reflect the complexity of dietary habits. Other measures, such as food frequency questionnaires combined with dietary records, have been suggested to obtain more accurate estimates of dietary intake than those obtained using individual methods [30].

In conclusion, we found support for a non-linear relationship between alcohol consumption and risk of diabetes in men and women from the general population. Our results further indicate that frequent consumption of alcohol is associated with the lowest risk of diabetes, even after taking average weekly alcohol consumption into account.

Acknowledgements We gratefully acknowledge the contribution of all study participants of the Danish National Health Examination Study.
Data availability In accordance with the Danish Act on Processing of Personal Data and the requirement for informed consent from the participants, study data cannot be made available in a public repository and cannot be obtained upon request.

Funding This research received no specific grant from any funding agency in the public, commercial or not-for-profit sectors. The collection of data to DANHES was funded by the Ministry of the Interior and Health and the Tryg Foundation.

Duality of interest The authors declare that there is no duality of interest associated with this manuscript.

Contribution statement $\mathrm{CH}$ contributed to the study design, the analysis and interpretation of the data, critical review and revision of the manuscript and drafted the original manuscript. UB contributed to the study design, the interpretation of the data, critical review and revision of the manuscript. MEJ contributed to the interpretation of the data and critical review and revision of the manuscript. MG contributed to the collection of data, study design, interpretation of the data and critical review and revision of the manuscript. JST contributed to the study design, the analysis and interpretation of the data and critical review and revision of the manuscript. JST is the guarantor of this work and had full access to all the data in the study and takes responsibility for the integrity of the data and the accuracy of the data analysis. All authors approved the final manuscript.

\section{References}

1. Athyros VG, Liberopoulos EN, Mikhailidis DP et al (2007) Association of drinking pattern and alcohol beverage type with the prevalence of metabolic syndrome, diabetes, coronary heart disease, stroke, and peripheral arterial disease in a Mediterranean cohort. Angiology 58:689-697

2. Beulens JW, van der Schouw YT, Bergmann MM et al (2012) Alcohol consumption and risk of type 2 diabetes in European men and women: influence of beverage type and body size the EPIC-InterAct study. J Intern Med 272:358-370

3. Clerc O, Nanchen D, Cornuz J et al (2010) Alcohol drinking, the metabolic syndrome and diabetes in a population with high mean alcohol consumption. Diabet Med 27:1241-1249

4. Baliunas DO, Taylor BJ, Irving H et al (2009) Alcohol as a risk factor for type 2 diabetes: a systematic review and meta-analysis. Diabetes Care 32:2123-2132

5. Carlsson S, Hammar N, Grill V (2005) Alcohol consumption and type 2 diabetes: meta-analysis of epidemiological studies indicates a U-shaped relationship. Diabetologia 48:1051-1054

6. Knott C, Bell S, Britton A (2015) Alcohol consumption and the risk of type 2 diabetes: a systematic review and dose-response metaanalysis of more than 1.9 million individuals from 38 observational studies. Diabetes Care 38:1804-1812

7. Koppes LL, Dekker JM, Hendriks HF, Bouter LM, Heine RJ (2005) Moderate alcohol consumption lowers the risk of type 2 diabetes: a meta-analysis of prospective observational studies. Diabetes Care 28:719-725

8. Heianza Y, Arase Y, Saito K et al (2013) Role of alcohol drinking pattern in type 2 diabetes in Japanese men: the Toranomon Hospital Health Management Center Study 11 (TOPICS 11). Am J Clin Nutr 97:561-568

9. Conigrave KM, Hu BF, Camargo CA Jr, Stampfer MJ, Willett WC, Rimm EB (2001) A prospective study of drinking patterns in relation to risk of type 2 diabetes among men. Diabetes 50:2390-2395 
10. Sato KK, Hayashi T, Harita N et al (2012) Relationship between drinking patterns and the risk of type 2 diabetes: the Kansai Healthcare Study. J Epidemiol Community Health 66:507-511

11. Hong SW, Linton JA, Shim JY, Kang HT (2015) High-risk drinking is associated with a higher risk of diabetes mellitus in Korean men, based on the 2010-2012 KNHANES. Alcohol 49:275-281

12. Huang J, Wang X, Zhang Y (2017) Specific types of alcoholic beverage consumption and risk of type 2 diabetes: a systematic review and meta-analysis. J Diabetes Investig 8:56-68

13. Koloverou E, Panagiotakos DB, Pitsavos C et al (2015) Effects of alcohol consumption and the metabolic syndrome on 10-year incidence of diabetes: the ATTICA study. Diabetes Metab 41:152-159

14. Wannamethee SG, Camargo CA, Manson JAE, Willett WC, Rimm EB (2003) Alcohol drinking patterns and risk of type 2 diabetes mellitus among younger women. Arch Intern Med 163:1329-1336

15. Marques-Vidal P, Vollenweider P, Waeber G (2015) Alcohol consumption and incidence of type 2 diabetes. Results from the CoLaus study. Nutr Metab Cardiovasc Dis 25:75-84

16. Cullmann M, Hilding A, Ostenson CG (2012) Alcohol consumption and risk of pre-diabetes and type 2 diabetes development in a Swedish population. Diabet Med 29:441-452

17. Carlsson S, Hammar N, Grill V, Kaprio J (2003) Alcohol consumption and the incidence of type 2 diabetes: a 20-year follow-up of the Finnish twin cohort study. Diabetes Care 26:2785-2790

18. Rasouli B, Ahlbom A, Andersson T et al (2013) Alcohol consumption is associated with reduced risk of type 2 diabetes and autoimmune diabetes in adults: results from the Nord-Trondelag health study. Diabet Med 30:56-64

19. Eriksen L, Gronbaek M, Helge JW, Tolstrup JS, Curtis T (2011) The Danish Health Examination Survey 2007-2008 (DANHES 2007-2008). Scand J Public Health 39:203-211
20. Carstensen B, Kristensen JK, Marcussen MM, Borch-Johnsen K (2011) The National Diabetes Register. Scand J Public Health 39: $58-61$

21. Saltin B, Grimby G (1968) Physiological analysis of middle-aged and old former athletes. Comparison with still active athletes of the same ages. Circulation 38:1104-1115

22. Schrieks IC, Heil AL, Hendriks HF, Mukamal KJ, Beulens JW (2015) The effect of alcohol consumption on insulin sensitivity and glycemic status: a systematic review and meta-analysis of intervention studies. Diabetes Care 38:723-732

23. Durrleman S, Simon R (1989) Flexible regression models with cubic splines. Stat Med 8:551-561

24. Green A, Sortso C, Jensen PB, Emneus M (2015) Validation of the Danish National Diabetes Register. Clin Epidemiol 7:5-15

25. Pietraszek A, Gregersen S, Hermansen K (2010) Alcohol and type 2 diabetes. A review. Nutr Metab Cardiovasc Dis 20:366-375

26. Naimi TS, Xuan Z, Brown DW, Saitz R (2013) Confounding and studies of 'moderate' alcohol consumption: the case of drinking frequency and implications for low-risk drinking guidelines. Addiction 108:1534-1543

27. Bahadoran Z, Mirmiran P, Azizi F (2013) Dietary polyphenols as potential nutraceuticals in management of diabetes: a review. $J$ Diabetes Metab Disord 12:43

28. Stockwell T, Zhao J, Panwar S, Roemer A, Naimi T, Chikritzhs T (2016) Do "moderate" drinkers have reduced mortality risk? A systematic review and meta-analysis of alcohol consumption and all-cause mortality. J Stud Alcohol Drugs 77:185-198

29. Feunekes GI, van't Veer P, van Staveren WA, Kok FJ (1999) Alcohol intake assessment: the sober facts. Am J Epidemiol 150: 105-112

30. Shim JS, Oh K, Kim HC (2014) Dietary assessment methods in epidemiologic studies. Epidemiol Health 36:e2014009 